\title{
El rol de Cuidador de personas dependientes y sus repercusiones sobre su Calidad de Vida y su Salud
}

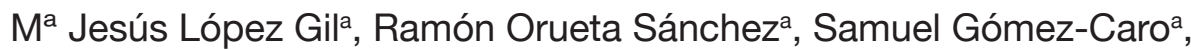 \\ Arancha Sánchez Oropesa ${ }^{a}$, Javier Carmona de la Morena ${ }^{a}$, Francisco Javier Alonso Moreno ${ }^{a}$
}

\begin{abstract}
a Médico de Familia. Centro de Salud "Sillería" (Toledo)
\end{abstract}

\section{Correspondencia:}

$M^{a}$ Jesús López Gil,

C/ Ribeiro $n^{\circ} 4,4^{\circ} \mathrm{C}$,

28915-Madrid.

Telf.: 645128615.

Recibido el 17 de febrero de 2009.

Aceptado para su publicación el 10 de mayo de 2009.

\section{RESUMEN}

Objetivo. Conocer la sobrecarga sentida por los cuidadores y las repercusiones que este rol representa sobre su calidad de vida, su salud en las esferas física, psíquica y social y su necesidad de utilización de los recursos sanitarios (frecuentación).

Diseño. Estudio observacional y analítico de casos y controles

Emplazamiento. Centro de salud de características urbanas.

Participantes. El grupo de estudio fue formado por 156 cuidadores principales y el grupo control por 156 personas adscritas al centro de salud que no realizaban funciones de cuidador principal, emparejadas por edad, sexo y número de patologías crónicas padecidas.

Mediciones. Características clínicas y sociodemográficas, datos relativos al rol de cuidador (parentesco, tiempo de desempeño del rol de cuidador, etc.), sobrecarga del cuidador (escala de Zarit), frecuentación, salud percibida, calidad de vida subjetiva (cuestionario de salud de Nottingham), presencia de sintomatología, ansiedad y depresión (EADG-18), función familiar (APGAR familiar y apoyo social percibido (cuestionario de Duke-UNC).

Resultados. El 66,4\% de los cuidadores presentaba sobrecarga en el test de Zarit. El 48,1\% los cuidadores respondió que su salud era mala o muy mala, frente al $31,4 \%$ del grupo control $(\mathrm{OR}=2,02$; IC 95\%: 1,27 - 3,21). La frecuentación media en el último año fue de 8,37 visitas/año en el grupo de cuidadores y de 7,12 visitas/año en el grupo control $(p<0,01)$. Se observó una mayor prevalencia de ansiedad y depresión en el grupo de cuidadores, así como una mayor frecuencia de posible disfunción familiar y de sensación de apoyo social insuficiente.

Conclusiones. Los resultados indican la necesidad de actuaciones multidisciplinarias encaminadas a apoyar a los cuidadores tanto de forma preventiva como de soporte. Con dichas intervenciones se han obtenido resultados positivos en diversos aspectos relativos tanto a la persona atendida como al cuidador, siendo necesario adaptar las intervenciones a las características y necesidades específicas de cada cuidador.

Palabras clave. Cuidadores, Calidad de Vida.

\section{ABSTRACT}

The caregiver burden and its repercussions on quality of life and health

Objective. To determine caregiver burden and its repercussions on quality of life, physical and mental health and social life and the use for healthcare resources (frequency).

Design. Observational, analytical, case control study.

Setting. Primary Health Care Centre in an urban setting.

Participants. The case group was made up of 156 primary caregivers and the control group of 156 personas assigned to the Primary Health Care Centre who were not primary care givers. They were paired by age, gender and number of chronic diseases.

Measurements. Clinical and socio-demographic characteristics, caregiver role characteristics (kinship, time performing role of caregiver, etc.), caregiver burden (Zarit scale), frequency, perceived health, subjective quality of life (Nottingham Health Profile), presence of anxiety and depression symptoms (GADS-18), family functioning (Family APGAR) and perception of family and social support (DukeUNC questionnaire).

Results. According to the Zarit scale, burden was present in $66.4 \%$ of caregivers. Compared to $31.4 \%$ of persons in the control group, $48.1 \%$ of caregivers considered their health was poor or very poor $(\mathrm{OR}=2.02 ; 95 \% \mathrm{Cl}: 1.27-3.21)$. The mean frequency in the last year was 8.37 visits/year in the caregiver group and 7.12 visits/year in the control group $(p<0.01)$. A higher prevalence of anxiety and depression was observed in the caregiver group, and a greater frequency of possible family dysfunction and sensation of insufficient social support.

Conclusions. The results indicate the need for multidisciplinary supportive and preventive interventions for caregivers. Such interventions have obtained positive results in several aspects both for the caregiver and the person cared for person. These interventions need to be adapted to the specific characteristics and needs of each caregiver.

Key words: Caregivers, Quality of Life. 


\section{INTRODUCCIÓN}

El aumento de la esperanza de vida en los países industrializados está produciendo un aumento de la población anciana en los mismos y esta población se caracteriza por sufrir un aumento de las patologías crónicas (osteoartrosis, insuficiencia cardiaca, etc.) lo que conlleva un declive físico y psíquico que condiciona su capacidad funcional, siendo cada vez mayor el número de personas que precisan de cuidados ${ }^{1-5}$. Se entiende por persona dependiente aquélla que por motivos de edad, enfermedad o discapacidad, y ligadas a la pérdida de autonomía física, sensorial, mental o intelectual, precisa con carácter permanente la atención de otra persona o ayuda para realizar las actividades básicas de la vida diaria ${ }^{6}$.

Gran parte de los cuidados precisados por estas personas recaen sobre los denominados cuidadores informales, entre los que destacan los cuidados prestados por la familia, que es el principal proveedor de cuidados de salud ${ }^{7-8}$. Así, la labor de los cuidadores informales contribuye al mantenimiento de las personas en su entorno social, disminuyendo la utilización de recursos formales y demorando o evitando el ingreso en instituciones ${ }^{9-10}$.

La presencia de un miembro que precisa de cuidados, genera una nueva situación familiar que puede provocar importantes cambios dentro de la estructura familiar y en los roles y patrones de conducta de sus integrantes. Estos cambios pueden precipitar crisis que ponen en peligro la estabilidad de la familia, pudiendo afectar a todos sus componentes, especialmente al cuidador principal, que es el miembro de la familia que soporta la mayor parte de la sobrecarga física y emocional de los cuidados ${ }^{11-12}$. Se define como cuidador principal informal (CPI) a la persona encargada de ayudar en las necesidades básicas e instrumentales de la vida diaria del paciente durante la mayor parte del día, sin recibir retribución económica por ello ${ }^{13-15}$.

La sobrecarga que soporta el cuidador, una vez superados los recursos disponibles, puede repercutir de forma negativa sobre su salud, siendo numerosos los trabajos publicados en los que se reflejan dichas repercusiones negativas, destacando por su frecuencia las referidas al malestar psíquico (principalmente ansiedad y depresión), aunque también se han descrito repercusiones importantes en otras esferas como la salud física, el aislamiento social, la falta de tiempo libre, la calidad de vida o el deterioro de la situación económica $8,13,16-20$, dando lugar a lo que algunos autores han dado en denominar síndrome del cuidador ${ }^{21-23 .}$

Por otra parte, existen descritas en la bibliografía evidencias sobre la eficacia de distintas intervenciones encaminadas a mejorar los cuidados a los pacientes y a aportar estrategias que permitan al cuidador afrontar este tipo de situaciones ${ }^{24-26}$.
Teniendo en cuenta todo lo referido, nos planteamos el presente trabajo, cuyos objetivos principales son conocer la sobrecarga sentida por los cuidadores y las repercusiones que este rol representa sobre su calidad de vida, su salud en las esferas física, psíquica y social y su necesidad de utilización de los recursos sanitarios (frecuentación).

\section{MATERIAL Y MÉTODO}

Se trata de un estudio observacional y analítico de casos y controles (figura 1) que fue realizado en el Centro de Salud "Sillería" (Toledo), centro de características urbanas que atiende a una población de aproximadamente 12.000 habitantes, los cuales presentan una pirámide de población característica de población en regresión (alto porcentaje de población anciana y bajo porcentaje de población infantil).

El grupo de estudio fue formado por cuidadores principales que llevasen al menos 2 meses realizando de forma ininterrumpida dicha función, considerándose como cuidador principal al encargado de ayudar en las necesidades básicas e instrumentales de la vida diaria del paciente durante la mayor parte del día, sin recibir retribución económica por ello ${ }^{13-15}$. Fueron criterios de exclusión no aceptar participar en el estudio, llevar menos de 2 meses realizando dicha función o percibir algún tipo de compensación económica por el desempeño de la misma.

El grupo control se formó con población adscrita al Centro de Salud que no realizaba función de cuidador principal y ajustada al grupo de estudio por edad, sexo y número de patologías crónicas padecidas, siendo elegido un control por cada cuidador incluido en el estudio (inicialmente se asignaron dos controles para cada caso, seleccionándose el segundo en caso de rechazo a participar del primero).

Con cada integrante de ambos grupos se concertó una entrevista individualizada en el Centro de Salud o en su propio domicilio. Dicha entrevista fue realizada por el médico de familia o por el residente de medicina familiar y comunitaria de último año de formación adscrito al cupo médico, y en ella se cumplimentó un cuestionario predefinido que fue complementado con información recogida de su historia clínica. Las variables recogidas a través del cuestionario y/o de la historia clínica fueron:

- Datos sociodemográficos: edad, sexo, nivel cultural, trabajo o no fuera del domicilio y personas que conviven en el mismo domicilio.

- Datos clínicos (obtenidos de la historia clínica): número de patologías crónicas padecidas y número de fármacos consumidos.

- Datos relativos al rol de cuidador: parentesco con la persona cuidada, tiempo que lleva desempeñando dicho rol y la existencia o no de otros cuidadores. - Sobrecarga del cuidador: evaluada a través de la es- 
cala de Zarit, la cual es considerada como la más adecuada para esta finalidad ${ }^{27}$ y consta de 22 ítems con una puntuación entre 1 y 5 puntos cada uno. Existe una versión validada en castellano y permite establecer la existencia de sobrecarga leve con puntuaciones entre 47 y 55 puntos y de sobrecarga intensa con puntuaciones iguales o superiores a 56 puntos $^{28-29}$.

- Frecuentación (dato obtenido de la historia clínica): número de consultas realizadas en los últimos 12 meses en su Centro de Salud, tanto a demanda como programadas, en el propio centro o en el domicilio del paciente, e incluyendo tanto las visitas médicas como las visitas a la consulta de enfermería. Se consideró como hiperfrecuentador aquél que consultó en quince o más ocasiones a lo largo del periodo indicado.

- Salud percibida: se investigo a través de una escala de Likert que con la pregunta "¿Cómo considera su estado de salud?" permitía 5 opciones (muy buena, buena, regular, mala y muy mala).

- Calidad de vida subjetiva: se investigó a través del cuestionario de salud de Nottingham (Nottingham Health Profile -NHP-), que consta de 38 ítems y presenta una valoración global y de 6 esferas especificas (energía, dolor, movilidad, sueño, estado emocional y relaciones sociales), también validado en castellano $30-32$

- Presencia de sintomatología: se empleo un cuestionario con preguntas cerradas donde estaban incluidos varios síntomas (apatía, nerviosismo, alteración del sueño, cansancio, pesadez de piernas, dolores musculares, dolor de espalda y cefalea) y también con preguntas abiertas para poder reflejar otros síntomas padecidos.

- Ansiedad y depresión: para evaluar la presencia de estos procesos se utilizó la escala de ansiedad-depresión de Golberg (EADG-18), cuestionario que presenta adecuados índices de validez, adaptado al castellano y ampliamente utilizado desde hace algunas décadas ${ }^{33-35}$.

- Función familiar percibida: se interrogó sobre la percepción del cuidador acerca de la función/disfunción familiar en su núcleo mediante el test de APGAR familiar, cuestionario que consta de 5 ítems y esta validado en castellano ${ }^{36-37}$.

- Apoyo social percibido: para valorar la sensación de apoyo social percibida por el cuidador se empleó el cuestionario de apoyo social de Duke-UNC, el cual consta de 11 ítems con cinco respuestas posibles en cada uno, también validado en nuestro idioma ${ }^{38-40}$.

Una vez recogidos los datos, estos fueron introducidos en una base de datos creada a tal fin en el programa estadístico R-SIGMA. Para el análisis de los mismos se emplearon herramientas de estadística descriptiva básica (rango, medía aritmética, desviación estándar, etc.) y analítica (t de Student, Chi-cuadrado, etc.). Así mismo, para la comparación de grupos se empleó la odds-ratio (OR) con intervalos de confianza al 95\%.

\section{RESULTADOS}

En total fueron incluidas 156 personas cuidadoras y 156 personas en el grupo control. Los cuidadores

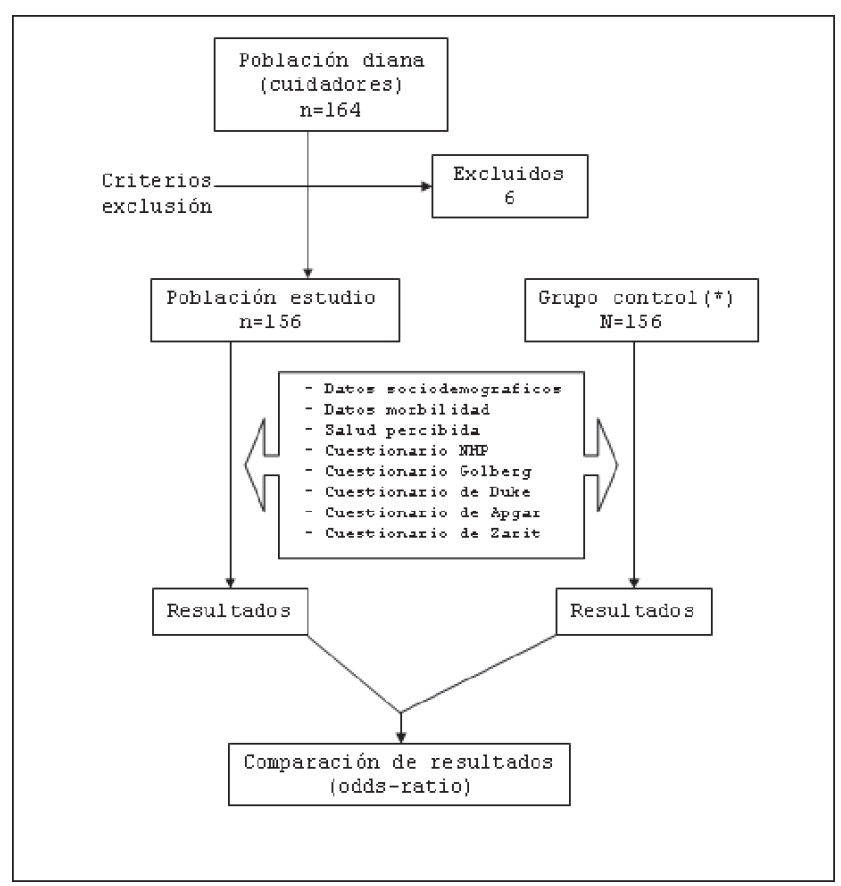

Figura 1. Esquema del Estudio.

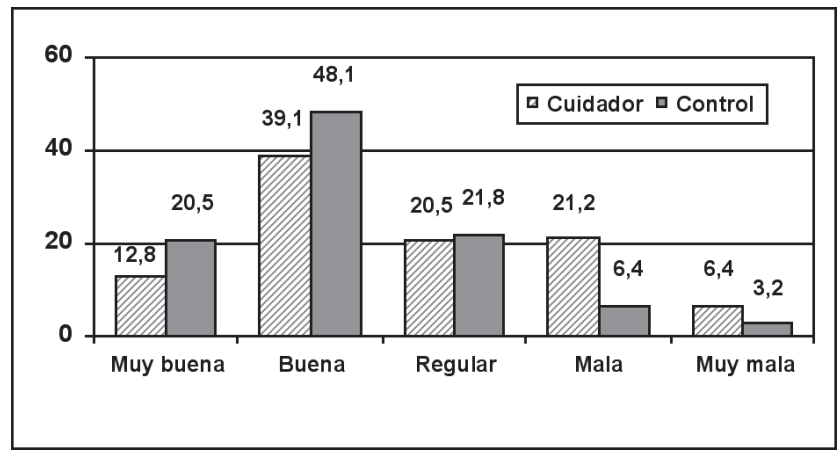

Figura 2. Salud percibida (cuidador vs control).

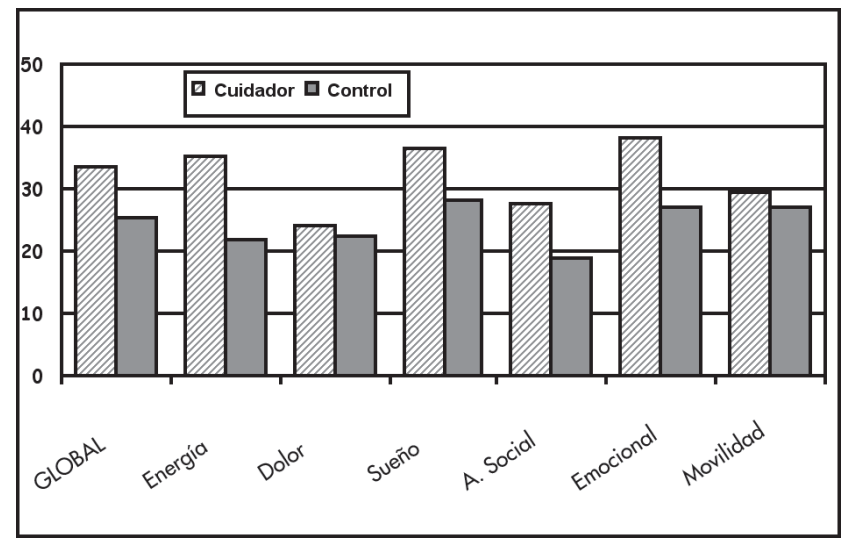

Figura 3. Calidad de vida (cuidador vs control). 

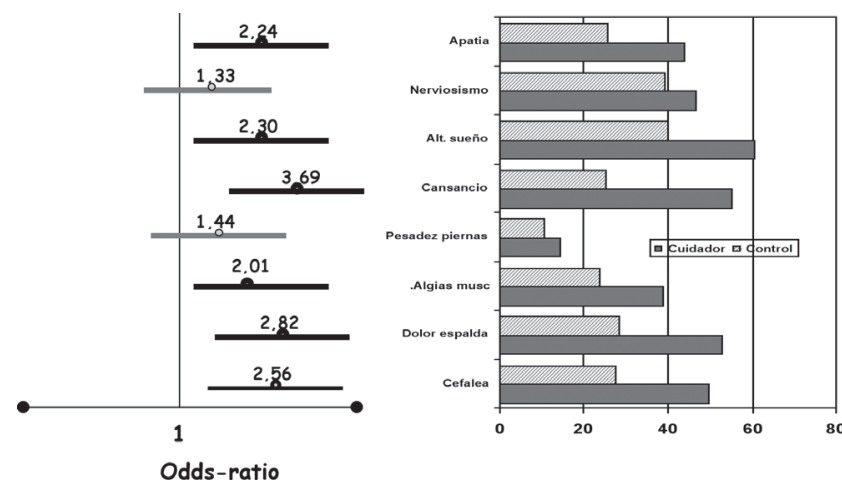

Figura 4. Síntomas (cuidador vs control).

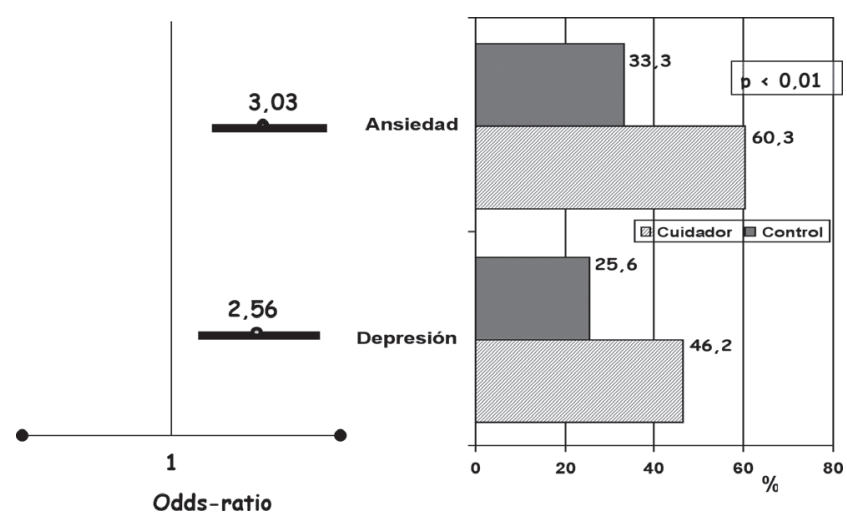

Figura 5. Ansiedad/depresión (cuidador vs control).
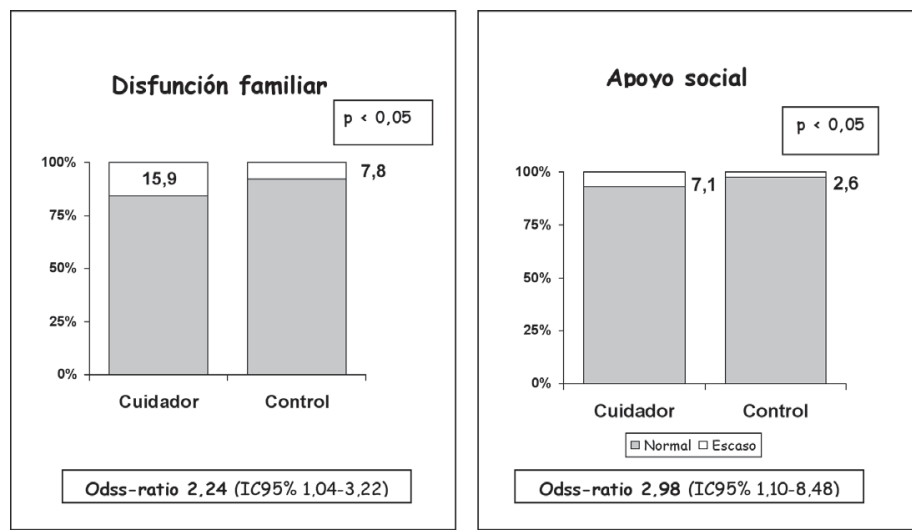

Figura 6. Disfunción familiar y apoyo social (cuidador vs control).

tenían una edad media de 57,6 años (IC 95\%: 48,4 66,8 años) y un rango de de edad entre 27 y 83 años, siendo el $87,8 \%$ mujeres. Presentaban un número medio de enfermedades crónicas de 2,7 (IC 95\%: 0,5 - 4,9). Estos datos fueron, al ser estos los criterios de selección, similares en el grupo control.

En el $84 \%$ de los casos convivía algún familiar más y el $53,2 \%$ eran cuidadores únicos. El tiempo medio como cuidador era de 4,2 años ( $\pm 3,9$ años).

El $66,4 \%$ de los cuidadores presentaba sobrecarga en el test de Zarit, siendo leve-moderada en el $23,7 \%$ y severa en el $32,7 \%$. La puntuación media obtenida en dicho cuestionario fue de 53,7 puntos (IC 95\%: $47,6-59,9)$.

Respecto a la salud percibida, la distribución de las respuestas obtenidas en ambos grupos aparece recogida en la figura 2. Agrupando los distintos ítems, el $48,1 \%$ los cuidadores respondió que su salud era mala o muy mala, frente al $31,4 \%$ del grupo control $(\mathrm{OR}=2,02 ; \mathrm{IC} 95 \%$ : 1,27 - 3,21). Valorada su calidad de vida subjetiva a través de las respuestas a las distintas preguntas del cuestionario NHP, los resultados (porcentaje de deterioro) tanto en la puntuación global como en los distintos apartados de dicho cuestionario aparecen recogidos en la figura 3 , existiendo una peor calidad de vida, estadísticamente significativa, en los cuidadores en la valoración global y en las esferas de energía, sueño, relaciones sociales y emocional.

La frecuentación media en el último año fue de 8,37 visitas/año en el grupo de cuidadores y de 7,12 visitas/año en el grupo control $(p<0,01)$. Fue hiperfrecuentador (más de 15 visitas/año) el $21,2 \%$ de los cuidadores, frente al $11,6 \%$ del grupo control $(\mathrm{OR}=$ 2,06; IC 95\%: 1,10 - 3,84).

La figura 4 muestra la presencia de distintos síntomas en ambos grupos y la odds-ratio de cada uno de los mismos. El grupo de cuidadores presentaba de forma significativa con mayor frecuencia los siguientes síntomas: cefalea, dolor de espalda, algias musculares, cansancio/fatiga, alteraciones del sueño y apatía.

Respecto a la esfera psíquica, la figura 5 presenta la prevalencia de ansiedad y depresión detectadas en ambos grupos a través del cuestionario de Golberg, existiendo mayor prevalencia de ambos cuadros en el grupo de cuidadores (OR = 3,03; IC 95\%: 1,91-4,81 y $\mathrm{OR}=2,49$; IC 95\%: 1,54 - 4,01, respectivamente).

La figura 6 muestra los datos relativos a la posible disfunción familiar en ambos grupos detectada a través del cuestionario de Apgar familiar, existiendo mayor porcentaje de disfunción en el grupo de cuidadores (15,9\% vs. $7,8 \%$; OR = 2,24, IC 95\%: 1,04-4,22). 
Por ultimo, la figura 6 también muestra los datos de ambos grupos relativos al apoyo social percibido a través del cuestionario de Duke-UNC, existiendo de forma significativa mayor sensación de apoyo insuficiente en el grupo de cuidadores $(7,1 \%$ vs. $2,6 \%$; OR = 2,98, IC 95\%: 1,10 - 8,48).

\section{DISCUSIÓN}

El presente estudio ha permitido aproximarnos a las características sociodemográficas del CPI que tiene a su cargo un enfermo dependiente, así como al impacto que el desempeño de dicho rol ocasiona sobre su salud física, psíquica y social y sobre la percepción de su calidad de vida. Nuestro estudio representa una importante aportación sobre el tema respecto a la mayoría de los publicados anteriormente, ya que la existencia de un grupo control permite, además de conocer la frecuencia de determinados síntomas o procesos, saber qué parte de los mismos puede atribuirse a la circunstancia de cuidador.

Dado que el estudio ha sido realizado en la población atendida en un Centro de Salud urbano de tamaño medio, la generalización de sus resultados y conclusiones debe ser realizada con precaución, aunque creemos que son representativos y generalizables, pues las características de nuestros cuidadores son similares a las descritas en otros grupos estudiados en la bibliografía. Así, el perfil del cuidador corresponde al de una mujer de edad media (40-60 años), ama de casa (sin trabajo fuera del domicilio), cónyuge o descendiente de primer grado de la persona cuidada (principalmente hija), con un nivel de estudios básicomedio y con varios años (entre 2 y 8) en el desempeño de la función de cuidador ${ }^{10,14,19,41-46}$.

Aunque en el momento actual existe una versión reducida de 7 ítems de la escala de Zarit para valorar la sobrecarga del cuidador ${ }^{47-48}$, en nuestro estudio utilizamos la escala de 22 ítems, puesto que es la empleada en la mayoría de los estudios publicados, lo que permite su comparación. El valor medio de sobrecarga encontrado en nuestros cuidadores es de 53,7 , que corresponde a un grado de sobrecarga leve-moderado y a un porcentaje de cuidadores con sobrecarga del $56,4 \%$, datos que, aunque llamativos, son concordantes, e incluso inferiores, a los encontrados en la bibliografía consultada ${ }^{10,29,42,44,46,49,50}$. Distintos estudios han permitido correlacionar la presencia de sobrecarga con factores clínicos y asistenciales, siendo los más importantes la edad y la vulnerabilidad clínica del cuidador y el grado de deterioro funcional y cognitivo del paciente atendido ${ }^{10,17,25,51-57}$. Conocer el grado de sobrecarga del CPI es importante porque está demostrada su relación con la calidad de vida del mismo ${ }^{14}$ y porque existen evidencias de que puede verse mitigado a través de diferentes intervenciones $^{10,17,25,50,55-57}$, siendo los cuidadores que soportan mayor grado de sobrecarga los que potencialmente más se beneficiarían de dichas actuaciones ${ }^{10}$.

Los resultados encontrados en nuestro estudio ponen en evidencia la vulnerabilidad del CPI a padecer en mayor medida problemas físicos y psíquicos, lo que determina la aparición del denominado síndrome del cuidador ${ }^{21-23,58}$, relacionándose incluso con un aumento de la mortalidad ${ }^{16}$. Con respecto a los síntomas físicos, referidos en mayor proporción que las personas incluidas en el grupo control, los datos coinciden con otras publicaciones, al destacar la mayor presencia de cansancio, dolor de espalda, cefalea, y algias musculares ${ }^{14,19,45,59,60}$.

Con referencia a la esfera psíquica, nuestros datos confirman el aumento de procesos como ansiedad y depresión y de síntomas como alteraciones del sueño, apatía o irritabilidad en el grupo de cuidadores respecto a la población de referencia, siendo incluso superiores a los encontrados en algunos trabajos previos $^{14,19,41,43,45,59,61}$. Hay que destacar que en nuestro estudio no encontramos un aumento significativo de síntomas como el nerviosismo, pero creemos que esto fue debido a que no fue un síntoma bien definido por nuestra parte, pudiendo no haberse entendido correctamente, ya que el cuestionario de Golberg sí detectaba un aumento significativo de la presencia de ansiedad respecto al grupo control. Algunos estudios han relacionado un mayor deterioro en la esfera psíquica del cuidador con la prolongación del tiempo desempeñando este rol, con la mayor dependencia de la persona atendida, con la mayor sensación de sobrecarga y con la menor presencia de apoyo social ${ }^{19,62-64}$.

El cuestionario de Duke-UNC pone de relieve que la mayoría de los cuidadores perciben el apoyo social que reciben como suficiente, si bien el pequeño porcentaje que lo percibe como insuficiente es estadísticamente superior al encontrado en el grupo control $(7,1 \%$ vs. $2,6 \%)$ y en consonancia con el encontrado en otras investigaciones ${ }^{43,65}$, todo ello pese a que es conocida que la inmensa mayoría de los cuidados recaen sobre los cuidadores informales ${ }^{8,66,67}$. Creemos que la percepción más o menos generalizada de tener un apoyo social suficiente no se ajusta a la realidad, sino que puede estar relacionada con que un alto porcentaje de cuidadores son mujeres que no trabaja fuera del domicilio, lo que facilita el desempeño de dicho rol. Cultural y socialmente la sociedad mantiene en general estrechos vínculos familiares que implican la atención a los miembros dependientes ${ }^{65,68-71}$, pero la sociedad esta experimentando cambios en los modelos familiares que dificultan asumir este tipo de funciones ${ }^{72-73}$.

La elevada presencia de problemas de salud entre nuestros CPI se refleja también en su calidad de vida. El cuestionario de calidad de vida de Nottinghan pone de manifiesto que nuestros cuidadores presentan ma- 
yor deterioro que el grupo control, tanto a nivel global como en las esferas de energía, sueño, emocional y de apoyo social. Aunque empleando distintas escalas y cuestionarios, otros investigadores han encontrado resultados comparables a los nuestros que muestran, en general, una relación directa entre el grado de sobrecarga expresado y la peor calidad de vida subjetiva ${ }^{14,74-78}$. En la misma línea estaría el elevado porcentaje de cuidadores que percibe su salud como "mala" o "muy mala", muy superior al encontrado en el grupo control.

Aunque es conocido que con relativa frecuencia la realización de cuidados informales en el seno de una familia supone cambios de roles en sus miembros y que esto puede ocasionar situaciones de disfunción $^{8,79}$, la mayoría de las investigaciones no abordan este aspecto cuando estudian las consecuencias de ser cuidador. En nuestro estudio hemos encontrado un porcentaje de familias disfuncionantes del 15,9\% en el grupo de cuidadores, cifra superior a la existente en el grupo control $(7,8 \%)$ y también superior al de alguna investigación que aporta este dato ${ }^{43}$.

Por último, al analizar la frecuentación de los servicios sanitarios por parte de los cuidadores, nuestros datos muestran una mayor frecuentación de los cuidadores respecto al grupo control, dato sobre el que existe gran diversidad en los distintos estudios publicados ${ }^{19,45,59,61}$. La mayor utilización de los servicios sanitarios encontrada en nuestro estudio podría justificarse por la mayor presencia de alteraciones en las esferas física y psíquica y por la peor calidad de vida percibida, considerando algunos autores que la mayor frecuentación no está en relación con la función de cuidador sino con los procesos padecidos ${ }^{19,61}$.

Todo lo anterior pone en evidencia la necesidad de implementar actuaciones multidisciplinarias encaminadas a apoyar a los cuidadores tanto de forma preventiva como de soporte, máxime cuando las revisiones realizadas sobre el tema muestran el beneficio potencial de las mismas ${ }^{24-26}$. Existen experiencias publicadas sobre diferentes tipos de intervenciones (formativas, psicoconductuales, grupos de apoyo, de descanso periódico, etc.) realizadas por distintos profesionales (médicos, enfermeras, psicólogos, equipos multidisciplinares, etc.). Con dichas intervenciones se han obtenido resultados positivos en diversos aspectos relativos tanto a la persona atendida como al cuidador (retraso en la institucionalización del paciente, disminución de la sobrecarga percibida por parte del cuidador y de las repercusiones que dicha sobrecarga suponía para las distintas esferas de la salud, etc) ${ }^{17,82-}$ ${ }^{89}$, siendo necesario adaptar las intervenciones a las características y necesidades específicas de cada cuidador ${ }^{90-91 .}$

\section{BIBLIOGRAFÍA}

1. United Nations. Population Division. World Population Ageing 1950-2050. WHO; 2002.
2. Instituto Nacional de Estadística. Tendencias demográficas durante el siglo XX en España. Madrid: INE; 1994.

3. Otero A, Zunzunegui MV, Rodríguez A, Aguilar MD, Lázaro P. Volumen y tendencias de la dependencia asociada al envejecimiento de la población española. Rev Esp Salud Pública 2004; 78:201-213

4. Romero L, Martín E, Navarro JL, Luengo C. El paciente anciano: demografía, epidemiología y utilización de recursos. En: Sociedad Española de Geriatría y Gerontología. Tratado de geriatría para residentes. Madrid: Marketing \& Comunication; 2006. p. 33-46.

5. Abellán A, Puga MD. Estudio monográfico: La discapacidad en la población española. En: Observatorio de personas mayores, editor. Las personas mayores en España. Informe 2004. Madrid: Ministerio de Trabajo y Asuntos Sociales; 2004. p. 777-809.

6. Ley $39 / 2006$, de 14 de diciembre, de Promoción de la Autonomía Personal y Atención a las personas en situación de dependencia. BOE 15 de Diciembre de 2006.

7. INSERSO. Cuidados en la vejez. El apoyo informal. Madrid: Ministerio de Asuntos Sociales. INSERSO; 1995.

8. García-Calvente MM, Mateo I, Gutiérrez, P. Cuidados y cuidadores en el sistema informal de salud. Granada: Escuela Andaluza de Salud Pública e Instituto Andaluz de la Mujer; 1999.

9. Davies B, Chekryn J, Martens M. Family functioning and its implications for paliative care. J Paliative Care 1994; 10:29-36.

10. Alonso A, Garrido A, Díaz A, Casquero R, Riera M. Perfil y sobrecarga de los cuidadores de pacientes con demencia incluidos en el programa ALOIS. Aten Primaria 2004; 33:61-68.

11. Olsen $\mathrm{H}$. The impact of serious illness on the family system. Postgraduate Medicine 1979; 47:169-174.

12. Puig C, Hernández Monsalve LM, Gérvas JJ. Los enfermos crónicos recluidos en su domicilio y su repercusión en la familia: una investigación cualitativa. Aten Primaria 1992; 10:550-552.

13. Ribas J, Castel A, Escalada B, Ugas L, Grau C, Magarolas L et al. Trastornos psicopatológicos del cuidador principal no profesional de pacientes ancianos. Rev Psiquiatría Fac Med Barna 2000; 27:131-134.

14. Badia X, Lara N, Roset M. Calidad de vida, tiempo de dedicación y carga percibida por el cuidador principal informal del enfermo de Alzheimer. Aten Primaria 2004; 34:170-177.

15. Lago Canzobre G, Debén Sánchez M. Cuidados del cuidador en el paciente con demencia. Disponible en: www.fisterra.com/ guias2/cuidador.htm.

16. Schulz R, Beach SR. Caregiving as a risk factor for mortality: the Caregiver Health Effects Study. JAMA 1999; 282:2215-2219.

17. Hepburn KW, Tornatore J, Center B, Ostwald SW. Dementia family caregiver training: affecting beliefs about caregiving and caregiver outcomes. J Am Geriatr Soc. 2001; 49:450-457.

18. Douglas SL, Baly BJ, Kelley CG, O'Toole E, Montenegro H. Impact of a disease management program upon caregivers of chronically critically ill patients. Chest 2005; 128:3925-3936.

19. López O, Lorenzo A, Santiago P. Morbilidad en cuidadores de pacientes confinados en su domicilio. Aten Primaria 1999; 24:404-410.

20. Roca M, Úbeda I, Fuentelsaz C, López R, Pont A, García L et al. Impacto del hecho de cuidar en la salud de los cuidadores familiares. Aten Primaria 2000; 26:53-67.

21. Schulz R, O’Brien AT, Bookwala J, Fleissner K. Psychiatric and physical morbidity effects of dementia caregiving: prevalence, correlates, and causes. Gerontologist 1995; 35:771-791.

22. Baumgarten M, Hanley JA, Infante-Rivard C, Battista RN, Becher R, Gauthier S. Health of family members caring for elderly persons with dementia. Ann Intern Med 1994;.120:126-32.

23. Covinsky KE. The impact of serious illness on patient's families. JAMA 1994; 272:1839-1844.

24. Knight BG, Lutzky SM, Macofsky-Urban F. A meta-analytic review of interventions for caregiver distress: recommendations for future research. Gerontologist 1993; 33:240-248.

25. Zabalegui A, Navarro M, Cabrera E, Gallart A, Bardallo MD, Rodríguez $\mathrm{E}$ et al. Eficacia de las intervenciones dirigidas a cuidadores principales de personas dependientes mayores de 65 años: una revisión sistematica. Rev Esp Geriatr Gerontol 2008; 43:157-166. 
26. Torres MP, Ballesteros E, Sánchez PD. Programas e intervenciones de apoyo a los cuidadores informales en España. Gerokomos 2008; 19:9-15.

27. Deeken JF, Taylor KL, Mangan P, Yabroff KR, Ingham JM. Care for the caregivers: a review of self report instruments developed to meansure the burden, needs and quality of life of informa caregivers. J Pain Symptom Manage 2003; 26:922-953.

28. Zarit SH, Reever KE, Bach-Peterson J. Relatives of the impaired elderly: correlates of feelings of burden. Gerontologist 1980; 20:649-655.

29. Martin M, Salvado I, Nadal S, Miji LC, Rico JM, Lanz P et al. Adaptación para nuestro medio de la escala de sobrecarga del cuidador (caregiver burden interview) de Zarit. Rev Gerontol 1996; 6 338-346.

30. Hunt SM, McKenna SP, McEwen J, Williams J, Papp E. The Nottingham Health Profile: Subjetive health Status and medical consultations. Soc Sci Med 1981; 15:221-229.

31. Hunt SM, McEwen J. The development to a subjetive health indicator. Social Health Illnes 1980; 2:231-346.

32. Alonso J, Anto JM, Moreno C. Spanish version of the Nottingham Health Profile: translation and preliminary validity. Am J Public Health 1990; 80:704-708.

33. Goldberg DP, Hillier VF. A scaled version of the General Health Questionnaire. Psychological Medicine 1979; 9:139-145.

34. Goldberg D, Bridges K, Duncan-Jones P, Grayson D. Detecting anxiety and depression in general medical settings. BMJ 1988; 297:897-899.

35. Montón C, Pérez-Echevarría MJ, Campos R, García Campayo J, Lobo A. Escalas de ansiedad y depresión de Goldberg: una guía de entrevista eficaz para la detección del malestar psíquico. Aten Primaria 1993; 12:345-349.

36. Smilkstein G. The family APGAR: a proposal for a family function test and its use by physicians. J Fam Pract 1978; 6:12311239

37. Bellón JA, Delgado A, Luna del Castillo JD, Lardelli P. Validez y fiabilidad del cuestionario de función familiar APGAR-familiar. Aten Primaria 1996; 18:289-296.

38. Broadhead WE, Gehlbach SH, Degruy FV, Kaplan BH. The Duke-UNC functional social support questionnaire: measurement of social support in family medicine patients. Med Care 1988; 26:709-723.

39. De la Revilla L, Bailón E, De Dios Luna J, Delgado A, Prados MA Freitas L. Validación de una escala de apoyo social funcional para su uso en la consulta del médico de familia. Aten Primaria 1991; 8 688-692.

40. Bellón JA, Delgado A, De Dios J, Lardelli P. Validez y fiabilidad del cuestionario de apoyo social funcional Duke-UNC-11. Aten Primaria 1996; 18:17-31.

41. Gálvez J, Ras E, Hospital I, Vila A. Perfil del cuidador principal y valoración del nivel de ansiedad y depresión. Aten Primaria 2003; 31:144-145.

42. Artaso B, Goñi A, Biurrun A. Cuidados informales en la demencia: predicción de sobrecarga en cuidadoras familiares. Rev Esp Geriatr Gerontol 2003; 38:212-218.

43. Moral MS, Juan J, López MJ, Pellicer P. Perfil y riesgo de morbilidad psíquica en cuidadores de pacientes ingresados en su domicilio. Aten Primaria 2003; 32:77-87.

44. García R, Vila R, Muñoz R. Perfil y grado de sobrecarga del cuidador principal de pacientes inmovilizados en un centro de salud. Enfermería Comunitaria 2005; 2:16-19.

45. Seira MP, Aller A, Calvo A. Morbilidad sentida y diagnosticada en cuidadores de pacientes inmovilizados en una zona de salud rural. Rev Esp Salud Pública 2002; 76:713-721.

46. Moreno-Gaviño L, Bernabeu-Wittel M, Alvarez-Tello M, Rincón-Gómez M, Bohórquez-Colombo P, Cassani-Garza M et al. Sobrecarga sentida por la figura del cuidador principal en una cohorte de pacientes pluripatológicos. Aten Primaria 2008; 40:193-198.

47. Gort M, March J, Gómez X, de Miguel M, Mazarico S, Ballesté J. Escala de Zarit reducida en cuidados paliativos. Med Clin (Barc) 2005; 124:651-653.
48. Regueiro A, Pérez-Vázquez A, Gómara S, Ferreiro M. Escala de Zarit reducida para la sobrecarga del cuidador en Atención Primaria. Aten Primaria 2007; 39:185-188.

49. Dueñas E, Martínez MA, Morales B, Muñoz Claudia, Viáfara AS, Herrera JA. Síndrome del cuidador de adultos mayores discapacitados y sus implicaciones psicosociales. Colombia Médica 2006; 37(suplemento 1):31-38.

50. Gort AM, Mazarico S, Ballesté J, Barberá J, Gómez X, de Miguel M. Uso de la escala de Zarit en la valoración de la claudicación en cuidados paliativos. Med Clin (Barc) 2003; 121:132-3.

51. Rosenvinge $H$, Jones $D$, Judge $E$, Martín A. Demented and Chronic depressed patients attending a day hospital: estress experienced by carers. Int J Geriat Psychiatry 1998; 13:8-11.

52. Leinonen E, Korpisammal L, Pulkkinen L, Pukuri T. The comparison of burden between caregiving spouses of depressive and demented patients. Int J Geriat Psychiatry 2001; 16:387-93.

53. Collins $\mathrm{C}$, Jones R. Emotional distress and morbidity in dementia carers: a matched comparison of husbands and wives. Int $J$ Geriat Psychiatry 1997; 12:1168-73.

54. Mittelman MS, Ferris SH, Shulman E, Steinberg G, Levin B. A family intervention to delay nursing home placement of patients with Alzheimer's disease. JAMA 1996; 276:1725-31.

55. Zarit SH, Anthony CR, Boutselis M. Interventions with caregivers of dementia patients: comparison of two approaches. Psychology Aging 1987; 2:225-32.

56. Chiverton P, Caine ED. Education to assist spouses in coping with Alzheimers Disease: a controlled trial. J Am Geriatr Soc 1989; 37:593-8.

57. Whitlatch CJ, Zarit SH, Von Eye A. Efficacy of interventions with caregivers: a reanalysis. Gerontologist 1991; 31:9-14.

58. Thompson C, Briggs M. Support for carers of people with Alzheimer's type dementia. Cochrane Database Syst Rev 2000; (2):CD00454

59. Morales JA, Muñoz T, Bravo F, Iniesta C, Montero FA, Olmos MC. Problemas de salud de los cuidadores de enfermos incapacitados. Centro de Salud 2000; 8:714-718.

60. Valles MN, Gutiérrez V, Luquin AM, Martín MA, López F. Problemas de salud y sociales de los cuidadores de los pacientes con demencia. Aten Primaria 1998; 22:481-485.

61. Díez J, Redondo LM, Arroniz C, Giacchia A, Zabal CH, Salaberri A. Malestar psiquico en cuidadores familiares de personas confinadas en su domicilio. MEDIFAM 1995; 5:124-130.

62. Segura Noguera JM, Bastida Bastús N, Martí Guadaño N, Riba Romeva M. Los enfermos crónicos domiciliarios y su repercusión en los cuidadores principales. Aten Primaria 1998; 21:431 436.

63. Rubio ML, Sánchez A, Ibáñez JA, Galvez F, Martí N, Mariscal J. Perfil médico-social del cuidador crucial, ¿se trata de un paciente oculto? Aten Primaria 1995; 16:181-186.

64. Rubio C, Bosch E, Soler E, Romero A, Martín M, García M. Repercusión en el estado de animo del cuidador principal de los pacientes que precisan cuidadores (PAID). Medicina Paliativa 2007; 7(supl 1):20.

65. Crespo M. López J. El apoyo a los cuidadores de familiares dependientes en el hogar: desarrollo del programa "Como mantener su bienestar". Colección Estudios. Madrid: Ministerio de Trabajo y Asuntos Sociales; 2007.

66. Instituto Nacional de Estadística. Encuesta sobre Discapacidades, Deficiencias y Estados de Salud. Madrid: Instituto Nacional de Estadística; 1999.

67. Casado-Marín D. La atención a la dependencia en España. Gac Sanit 2006; 20(Supl 1):135-142.

68. García-Calvente MM, Mateo-Rodríguez I, Eguiguren AP. El sistema informal de cuidados en clave de desigualdad. Gac Sanit 2004; 18(Supl 1):132-139.

69. Lawton MP, Moss M, Kleban MH, Glicksman A, Rovine M. A two-factor model of caregiving appraisal and psychological well-being. J Gerontol 1991; 46:181-189.

70. Dellmann-Jenkins M, Blankemeyer M, Pinkard O. Incorporating the elder caregiving role into the developmental task of young adulthood. Int J Aging Hum Dev 2001; 52:1-18. 
71. Murray J, Schneider J, Benerjee S, Mann A. EUROCARE: a cross-national study of co-resident spouse carers for people with Alzheimer's disease: II-A qualitative analysis of the experience of caregiving. Int J Geriatr Psychiatry 1999; 14:662-667.

72. Durán MA. Los costes invisibles de la enfermedad. $2^{\mathrm{a}}$ ed. Bilbao: Fundación BBVA; 2002.

73. Bazo MT, Domínguez-Alcón C. Los cuidados familiares de salud en las personas ancianas y las políticas sociales. Reis 1996; 73:43-56.

74. Ubeda I, Buisac D, Lacarcel M, Roca M, Salas AM, López C, et al. Características y calidad de vida de los cuidadores informales de enfermos de sida. Enfermería Clínica 2006; 16:137-143.

75. Marco E. Calidad de vida del cuidador del hemipléjico vascular. Tesis doctoral. Facultad de Medicina. Universidad Autónoma de Barcelona; 2004.

76. Martín M, Ballesteros J, Ibarra N, Loizaga C, Serrano B, Larumbe $\mathrm{MJ}$ et al. Alzheimer's caregiver burden and pshychological distress. A neglected association in the assessment of dementias. Actas Esp Psiquiatr 2002; 30:201-206.

77. Coen RF, O'Byle CA, Coakley D, Lawlor BA. Individual quality of life factors distinguishing low-burden and high-burden caregivers of dementia patients. Dement Geriatr Cogn Disord 2002; 13:164-170.

78. Bell CM, Araki SS, Neumann PJ. The association between caregiver burden and caregiver health-related quality of life in Alzheimer disease. Alzheimer Dis Assoc Disord 2001; 15:129-136.

79. Medalie $\mathrm{H}$. The patient and family adjustment to chronic disease in the home. Disabil Rehabil 1997; 19:163-170.

80. Marriot A, Donaldson C, Terrier N, Burns A. Effectiveness of cognitive-behavioural family intervention in reducing the burden of care in carers of patients with Alzheimer's disease. $\mathrm{Br} \mathrm{J}$ Psychiatry 2000; 176:557-562.

81. Losada A, Izal M, Montorio I, Márquez M, Pérez G. Eficacia diferencial de dos intervenciones psicoeducativas para cuidadores de familiares con Alzheimer. Rev Neurología 2004; 38:701-708.

82. Bliss $\mathrm{J}$. What do informal carers need from district nursing services? Br J Community Nurs 2006; 11:251-256.
83. Rabinowitz YG, Mausbach BT, Coon DW, Depp C, Thompson LW, Gallagher-Thompson D. The moderating effect of selfefficacy on intervention response in women family caregivers of older adults with dementia. Am J Geriatr Psychiatry 2006; 14:642-649.

84. López J. Entrenamiento en manejo del estrés en cuidadores de familiares mayores dependientes: desarrollo y evaluación de la eficacia de un programa. Tesis Doctoral. Universidad Complutense de Madrid; 2005.

85. Mittelman MS, Roth DL, Haley WE, Zarit SH. Effect of a caregiver intervention on negative caregiver appraisals of behavior problem in patients with alzheimer's disease. J Gerontol Psychological Sciences 2004; 59:27-34.

86. Gallalgher-Thompson D, Lovett S, Rose J, McKibbin C, Coon D, Futterman A, Thompson LW. Impact of psychoeducational interventions on distressed family caregivers. J Clin Geropsychol 2000; 6 91-110.

87. McCurry SM, Gibbons LE, Logsdon RG, Vitiello M, Teri L. Training caregivers to change the sleep hygiene practices of patient with dementia. The NITE-AD project. J Am Geriatrics Soc 2003; 51:1455-1460.

88. Egbert N, Koch L, Coeling H, Ayers D. The role of social support in the family and community integration of right-hemisphere stroke survivors. Health Commun 2006; 20:45-55.

89. Gallalgher-Thompson D, Coon DW, Solano N, Ambler C, Rabinowitz Y, Thompson LW. Change in indices of distress among Latina and Caucasian female caregivers of elderly relatives with dementia: Site specific results from the Reach National Collaborative Study. Gerontologist 2003; 4:580-591.

90. López J, Crespo M. Intervenciones con cuidadores de familiares mayores dependientes: una revisión. Psicothema 2007; 19:72-80.

91. Losada M, Moreno-Rodríguez R, Cigarán M, Peñacoba C. Análisis de programas de intervención psicosocial en cuidadores de pacientes con demencia. Informaciones Psiquiatritas 2006; 1984:173-186. 